\title{
A CONTRIBUIÇÃO DA PSICOPEDAGOGIA NO ATENDIMENTO DE CRIANÇAS COM DEFICIÊNCIA EM UM CENTRO DE REABILITAÇÃO DE ALTA COMPLEXIDADE ${ }^{1}$
}

Maria dos Remédios Mendes Chaves Barreto, UNR ${ }^{2}$ Izabel Herika Gomes Matias Cronemberger, UNIFSA ${ }^{3}$ Maria Andreia da Nóbrega Marques,UNIFSA ${ }^{4}$

\section{RESUMO}

Através da aprendizagem, o sujeito é inserido, de forma mais organizada no mundo cultural e simbólico que incorpora a sociedade. Este artigo objetiva analisar a contribuição do atendimento psicopedagógico na reabilitação e habilitação de crianças com deficiência no Centro Integrado de reabilitação (CEIR), em Teresina - PI. Seus objetivos específicos são: demonstrar a aprendizagem sobre a perspectiva psicopedagógica, compreender o trabalho psicopedagógico em um Centro de Alta Complexidade e; citar estratégias utilizadas no atendimento psicopedagógico do CEIR. A metodologia foi baseada em um estudo bibliográfico e documental, de natureza qualitativa e abordagem descritiva e explicativa, de forma analítica com a inclusão de informações publicadas pelo CEIR. Conclui-se que a psicopedagogia cumpre relevante função, na socialização dos conhecimentos disponíveis, promovendo o desenvolvimento cognitivo do aprendiz e tornando-o mais independente e autônomo, considerando suas limitações.

Palavras-chave: Psicopedagogia. Deficiência. Centro de Reabilitação.

\section{INTRODUÇÃO}

A educação do século XXI vê as dificuldades de aprendizagens como um dos maiores desafios sociais, pois as crianças que as vivenciam encontram barreiras durante os processos de integração e socialização. Para a integração e socialização dessas crianças é necessário criar condições de interação como trabalhos em grupos e utilizando o lúdico, estimulando, assim, a participação e comunicação de todos. Essa

\footnotetext{
${ }^{1}$ Trabalho apresentado no Congresso Brasileiro Ciência e Sociedade (CBCS 2019), promovido pelo Centro Universitário Santo Agostinho, de 03 a 05 de outubro de 2019, em Teresina-PI.

${ }^{2}$ Mestranda em Educação pela Universidade Nacional de Rosário - UNR - Argentina. Terapeuta do Centro de Reabilitação e Habilitação - CEIR Email: psic.remediosbarreto@hotmail.com

${ }^{3}$ Doutora e Mestra em Políticas Públicas, pela Univesidade Federal do Pauí, Brasil. Professora do Centro Universitário Santo Agostinho, Brasil. Email: izabel herika@hotmail.com

${ }^{4}$ Doutora em Psicologia pela Universidade São Francisco, Brasil. Professora Adjunta Nível 1 da Universidade Estadual do Piauí. Professora do Centro Universitário Santo Agostinho, Brasil. Email: mandreiamarques@yahoo.com.br.
} 


\section{conghESSO CIENCIAESOCIEDADE

questão reforça a posição de Ferreira (2010, p. 61) referindo-se a "arte como um recurso indispensável para a formação do indivíduo dentro do contexto social em que se encontra". Realçando que a deficiência pode favorecer a existência de barreiras ao aprender e que a psicopedagogia tem a aprendizagem e suas dificuldades como seus objetos de estudo e intervenção.

Do ponto de vista de Weiss (2008), a psicopedagogia busca a melhoria das relações com a aprendizagem, assim como a melhor qualidade na construção da própria aprendizagem de alunos e educadores, sendo assim desvelou-se o problema: Qual a contribuição da psicopedagogia na reabilitação e habilitação de crianças com deficiência no Centro Integrado de reabilitação - CEIR, em Teresina - PI?

Os problemas de aprendizagem não desaparecem; no entanto, a criança pode aprender a compensar suas dificuldades. Quanto mais cedo for realizada a intervenção a criança poderá aprender a conduzir melhor sua dificuldade em aprender, pois para o psicopedagogo o que preocupa não é o que a criança faz e sim o que ela não é capaz de fazer. Esta pesquisa tem como objetivo geral: Analisar a contribuição do atendimento psicopedagógico na reabilitação e habilitação de crianças com deficiência no Centro Integrado de reabilitação - CEIR, em Teresina - PI e como objetivos específicos: Demonstrar a aprendizagem sobre a perspectiva psicopedagógica, compreender o trabalho psicopedagógico no Centro Integrado de Reabilitação - CEIR e citar estratégias utilizadas no atendimento psicopedagógico do CEIR.

A mesma justifica-se pela observação do psicopedagogo, enquanto profissional capacitado para diminuir as dificuldades de aprendizagem das crianças com deficiência que não estão progredindo no ambiente escolar. Muitas vezes essa dificuldade é percebida pela família ou professor que encaminha o aluno ao psicopedagogo em busca de uma investigação mais detalhada do caso, para que possa detectar o problema e traçar metas para superá-las.

A metodologia da pesquisa foi baseada em um estudo bibliográfico e documental, de natureza qualitativa e abordagem descritiva e explicativa, em que os resultados foram apresentados de forma analítica por meio de reflexões, baseando-se nas informações extraídas a partir de relatórios publicados pelo CEIR, para tanto 


\section{CONQEFESSOCIENCIAESOCIEDADE

obteve-se autorização formal. A pesquisa qualitativa responde a questões muito particulares, com um nível de realidade que não pode ser quantificado. Ou seja, ela trabalha com um universo de significados, o que corresponde a um espaço mais profundo das relações, dos processos e dos fenômenos que não podem ser reduzidos à operacionalização de variáveis (LÜDKE; ANDRÉ, 2014).

A pesquisa descrita apresenta-se estruturada em seções, onde inicialmente temos as notas introdutórias. Na segunda seção descrevemos sobre intervenção psicopedagógica, destacando a aprendizagem sobre a perspectiva psicopedagógica. $\mathrm{Na}$ terceira seção apresentamos a atuação psicopedagógica no Centro Integrado de reabilitação - CEIR, seguido das considerações finais.

\section{INTERVENÇÃO PSICOPEDAGÓGICA OLHAR QUE FAVORECE A INCLUSÃO}

A Psicopedagogia surgiu na Europa, em meados do século XIX, onde a Medicina, Psicologia e a Psicanálise, começaram a se preocupar com uma alternativa de intervenção nos problemas de aprendizagem (BOSSA, 2000, p.37). Os primeiros centros psicopedagógicos foram fundados na Europa (1946) por Boutonier e George Mauco, com direção médica e pedagógica unindo conhecimento na área da psicologia, psicanálise e pedagogia onde tentavam readaptar crianças com comportamentos socialmente inadequados na escola ou no lar, e atender crianças com dificuldades de aprendizagens apesar de serem inteligentes (BOSSA, 2000, p.39).

No Brasil, a psicopedagogia surge aproximadamente nos anos 70, a partir da necessidade de atendimento a crianças com distúrbios na aprendizagem, consideradas inaptas dentro do sistema educacional convencional, porém os cursos na área só começaram a se multiplicarem na década de 90. "A psicopedagogia tratava-se de uma área que utiliza determinados conhecimentos no sentido de dar suporte ao ego fragilizado do aluno e cujo o trabalho estaria mais voltado para as dificuldades escolares específicas do mesmo"(CHAMAT, 2014, p. 17). O psicopedagogo deve agir em situações onde o aluno não está tendo desempenho escolar favorável, promovendo assim ações preventivas e corretivas, para que estes alunos possam de certa maneira superar essas dificuldades e passar a melhorar seu desempenho escolar. 
Bossa (2000, p.23) afirma que: "cabe ao psicopedagogo saber como se constitui o sujeito, como este se transforma em suas diversas etapas de vida e quais os recursos de conhecimento de que ele dispõe pela forma pela qual produz conhecimento". O psicopedagogo pode trabalhar o lúdico com os aprendentes, incluindo as cores os desenhos, o giz de cera, massas para modelar, aquarela, blocos lógicos ou outros recursos que podem estar auxiliando o profissional a trabalhar em grupo a socialização e interação dessas crianças.

\begin{abstract}
A psicopedagogia tem por objetivo compreender, estudar e pesquisar a aprendizagem nos aspectos relacionados com o desenvolvimento e ou problemas de aprendizagem. A aprendizagem é entendida aqui como decorrente de uma construção, de um processo, o qual implica em questionamentos, hipótese, reformulações, enfim implica um dinamismo. A psicopedagogia tem como meta compreender a complexidade dos múltiplos fatores envolvidos neste processo (RUBINSTEN, 2001, p. 127).
\end{abstract}

A psicopedagogia vai em busca dos motivos que levam os indivíduos a não estarem alcançando os resultados necessários quanto a aprendizagem.O objetivo do psicopedagogo é fazer a intervenção psicopedagógica, ou seja, intervir de forma positiva no meio, quebrando um padrão existente anteriormente. Essas intervenções psicopedagógicas complementam a formação do aluno com vista à autonomia e independência na escola e fora dela, traz também capacidade de aquisição de habilidades assim como fornece técnicas de organização, estruturação, repetições e treinamento. O psicopedagogo pode exercer sua função tanto no ambiente escolar como também pode estar fazendo acompanhamento do mesmo em uma clínica reforçando o aluno a sanar suas dificuldades.

Weiss (2008, p. 45), afirma que: "O exercício da psicopedagogia não é para quem quer; é, sobretudo para quem pode". A autora ressalta que não adianta termos somente a teoria e sim uma junção da teoria com a prática a fim de buscar bons resultados do seu trabalho, mostrando de maneira clara os seus benefícios junto às pessoas que buscam por uma ajuda psicopedagógica. Desse modo, o autor nos chama a atenção para o papel de cada um dentro da sociedade, pois não devemos nos acomodar ou nos adaptar diante de situações desfavoráveis, e assim realça-se o papel 


\section{CONQEFESSOCIENCIAESOCIEDADE

do psicopedagogo que busca incessantemente as causas do não aprender, ou seja, das dificuldades de aprendizagem.

É possível destacar alguns dos importantes benefícios que podem ser alcançados por meio das brincadeiras e dos jogos como veículos da aprendizagem, em que é possível ver o desenvolvimento de habilidades de coordenação motora e de linguagem. Sabe-se que para a criança tanto as brincadeira quanto os jogos são de fundamental importância para que haja o seu desenvolvimento, para que ela descubra coisas novas, que ela invente, e que pratique todas as suas habilidades, portanto, tanto os brinquedos quanto os jogos são elementos responsáveis para proporcionar e incentivar sua autoconfiança, que consequentemente se transformará em aprendizado sobre o desenvolvimento da linguagem, do modo de pensamento, e também de sua atenção e concentração. É possível também que desenvolva na mesma a capacidade de imitação, suas imaginações e memória, sendo também muito relevante para a sua saúde física, mental, intelectual, e conhecerão também o significado e o sentido da cooperatividade, da competição e, dessa forma estará mais apta a buscar novas alternativas para os problemas que surgirem em seu cotidiano.

Vygotsky (2001) afirma que: "a brincadeira é capaz de criar zonas de desenvolvimento proximal e que estas também proporcionam saltos qualitativos para o desenvolvimento e para a aprendizagem infantil". É com o uso das brincadeiras que será possível alcançarem-se resultados importantíssimos, pois há uma maior facilidade em desenvolver a aprendizagem. Diante disso, a criança estará conhecendo o mundo que a cerca, se socializando e construindo uma identidade com as brincadeiras e os jogos, e isso se explica por estas serem ferramentas fundamentais para o desenvolvimento psicológico e de grande valor psicopedagógico. É de fato o propulsor do desenvolvimento físico, mental e cognitivo, e formador de sua personalidade.

De acordo com Weiss (2008, p. 80): “o espaço lúdico durante o diagnóstico traz possibilidade de um melhor entendimento da real situação e nível de desenvolvimento do aprendente". Dessa forma, é cada vez mais nítido o valor agregado às ações lúdicas, como as brincadeiras e jogos educativos, que se expressam de maneira positiva como métodos psicopedagógicos de ensino-aprendizagem. São duas ferramentas que 


\section{congęESSO CIENCIAESOCIEDADE \\ Inovação, Diversidatle e Sustentahililitadle}

promovem um grande dinamismo na educação, e que garante bons resultados, visto que apresentam diversas possibilidades de metodologias de trabalho para os educadores, apresentando também várias formas de aprendizado aos alunos, capaz de instigar o desejo e o gosto pelo aprendizado.

\section{ATUAÇÃO PSICOPEDAGÓGICA NO CENTRO INTEGRADO DE REABILITAÇÃO - CEIR}

Conforme notícias publicitadas no site oficial do Centro Integrado de Reabilitação - CEIR, essa instituição foi inaugurada em 5 de maio de 2008, está localizado à Avenida Higino Cunha, no 1515, bairro Ilhotas, em Teresina-PI, atendendo a pessoas com deficiência físico-motora, auditiva e intelectual. É uma instituição consolidada como referência na saúde pública do Estado do Piauí. Conta com uma equipe multidisciplinar, com profissionais capacitados, técnicas modernas e equipamentos de última geração, com o diferencial de priorizar a humanização no atendimento.

O Centro oferece reabilitação com um atendimento multiprofissional que inclui serviços de fisioterapia, serviço social, fonoaudiologia, psicologia, psicopedagogia, arte-reabilitação, musicoterapia, fonoaudiologia, terapia ocupacional, musicoterapiae reabilitação desportiva. Além disso, tem uma equipede assistência clínica para atender às necessidades dos pacientes na cardiologia, neurologia, neuropediatria, ortopedia, pediatria, urologia, otorrinolaringologia, enfermagem, nutrição, dermatologia e fisiatria.

A reabilitação/habilitação física teve início com a inauguração do Centro. Em 2015, foi iniciada a reabilitação/habilitação intelectual que atende crianças com deficiência intelectual, Síndrome de Down e Transtornos do Espectro Autista (TEA), conhecido popularmente como autismo. Também teve início o Programa de Saúde Auditiva, que assiste pessoas com problemas auditivos nos graus leve, moderado, severo e profundo, com a concessão de aparelhos auditivos, o acompanhamento de por equipe multidisciplinar, e, a depender das demandas, a inclusão dessa pessoa na reabilitação/habilitação auditiva. 


\section{CONQBEESSOCIENCIAESOCIEDADE

Consta no site oficial da instituição e referência ao seu relatório de 2016 que nesse ano foi implantado no CEIR uma Clínica de Microcefalia, com a capacidade de atendimento para até 200 crianças com microcefalia relacionada ao Zika Vírus que devem ficar no Centro até, pelo menos, os três anos de idade recebendo estimulação precoce. Também dispõe de uma Oficina Ortopédica, com um parque industrial que confecciona e concede produtos ortopédicos sob medida e personalizados, e de um moderno Centro de Diagnóstico, que realiza mais de 100 tipos de procedimentos e exames, com conforto e praticidade. Ressaltamos que todos esses serviços são realizados por meio do Sistema Único de Saúde (SUS).

O Centro é administrado pela Associação Reabilitar, uma instituição social sem fins lucrativos, e assiste pessoas com deficiência permitindo um acompanhamento integrado, múltiplo e completo de cada paciente atendido. O CEIR é uma referência em reabilitação porque acredita na reabilitação e na superação da pessoa com deficiência, valorizando suas potencialidades. O trabalho desenvolvido objetiva a reintegração na sociedade, no mercado de trabalho e na família. Por isso mesmo são oferecidos todos os tratamentos num único lugar, com profissionais capacitados, técnicas modernas e equipamentos de última geração, através de uma equipe multidisciplinar, onde são oferecidos os recursos clínicos para adaptação, readaptação e reabilitação à sua condição (RELATÓRIO CEIR, 2016).

Em relação ao eixo pedagógico direciona-se na ação educativo-terapêutica, tendo como base teórico- prática as concepções construtivistas (Piaget) e sóciointeracionistas (Vygotsky), além de várias outras contribuições importantes como as de Vitor da Fonseca, dentre outros.No trabalho clínico psicopedagógico do CEIR, busca-se não só compreender o porquê o paciente não aprender, mas também o que ele pode aprender e como. Esse trabalho psicopedagógico atende crianças com deficiência, com a sua multiplicidade de tipos e sequelas. Segundo Pain (2006), o trabalho do psicopedagogo é atuar na aprendizagem humana, diagnosticando causas de problemas nas variadas aprendizagens, dentre elas a escolar e intervindo, de forma específica e especializada com cada indivíduo em sua totalidade, promovendo o seu desenvolvimento integral, o que permite a ele construir o conhecimento. 


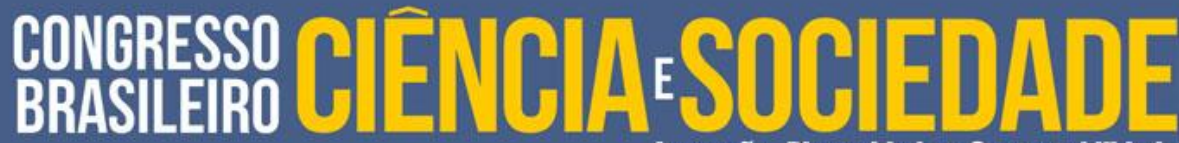

Para enveredarmos sobre o problema de aprendizagem, necessitamos primeiramente compreender o que é aprendizagem e como ela se processa no olhar psicopedagógico. Há na literatura vários modos de conceituar aprendizagem, muitos autores preocupam em definir o tema na visão psicopedagógica. Fernández (2001, p.57) relata que "todo sujeito tem sua modalidade de aprendizagem e os seus meios para construir o próprio conhecimento, e isso significa uma maneira muito pessoal para se dirigir e construir o saber". De acordo com o exposto pode-se tomar o processo cognitivo como um processo de formação da própria inteligência, e essa, como o conhecimento de uma capacidade geral de estabelecer relações, quer seja entre objetos, eventos ou situações. O autor faz correspondência entre construção da inteligência e processo de formação de conhecimentos de forma que os mais elementares servem de base para os mais complexos que se seguem.

“A aprendizagem é um fruto da história de cada sujeito e das relações que ele consegue estabelecer com o conhecimento ao longo da vida" (BOSSA, 2000, p.39). Porém, quando se fala em aprendizagem, não se pode relacionar o problema simplesmente à história do aprendente, pois, a aprendizagem não é um processo individual, ou seja, não depende só do esforço de quem aprende, mas sim de um processo histórico coletivo.

O trabalho no CEIR, frente ao atendimento psicopedagógico se fixa em três pilares: estimulação cognitiva, trabalhar as dificuldade de aprendizagem e praticar a inclusão, objetivando de forma geral levar a criança a (re) encontrar seu lugar de aprendiz na vida, família e escola e, especificamente, integrar os aspectos afetivo, cognitivo, social e perceptivos. Em relação à abordagem teórica, enfatiza a construção e reconstrução dos seus conceitos, a investigação, a elaboração de hipóteses e a troca de experiências, favorecendo o desenvolvimento global, através de atividades lúdicas.

Quantos aos procedimentos realizados na intervenção psicopedagógica no CEIR, esses dividem- se em três eixos, sendo: Eixo 1 - Avaliação pedagógica, que investiga o processo de aprendizagem e as dificuldades, estabelece estratégias, procedimentos e encaminhamentos necessários. Utiliza materiais como jogos, desenhos, livros de histórias, materiais pedagógicos diversos, etc; Eixo 2 - Orientações 


\section{conghESSO CIENCIAESOCIEDADE

escolares, em que busca proporcionar troca de experiências, inclusão e encaminhamentos; e Eixo 3 - Atendimento à criança que trabalha as dificuldades de aprendizagem, estimula a leitura, escrita e raciocínio lógico, e aborda o desenvolvimento intelectual e linguístico.

De acordo com Scoz (2008, p. 69) o papel da psicopedagogia, na qual o objeto de estudo é aprendizagem humana e suas características, inicialmente preocupa-se:

Com o processo de aprendizagem, onde a mesma estuda como se aprende, como se produz as alterações no processo de aprendizagem, como varia evolutivamente este processo, como reconhece as alterações da criança, como tratar estas dificuldades e como prevenir situações que podem interferir no processo ensinoaprendizagem. O trabalho do Psicopedagogo é fazer com que a criança se sinta responsável pela modalidade de querer conhecer e aprender (SCOZ, p. 69, 2008).

As dificuldades de aprendizagens variam, pois em cada um de nós existem pontos fortes e fracos em nosso processo de aprender e de adquirir conhecimentos, uns são melhores com a matemática outros na área das humanas, outros são ótimos com as artes, mas todos nós somos capazes de aprender. Para Fernandez (2001, p.76) "existem inúmeras causas das dificuldades de aprendizagem dentre elas a lesão cerebral", porém existem também outros influentes no processo de aprender e em suas dificuldades, não só as lesões cerebrais.

O atendimento psicopedagógico no CEIR ocorre em grupos realizando estimulação pedagógica e socialização das crianças, possibilitando também aos pais oportunidade para perceberem como estas respondem aos estímulos oferecidos e acompanhar o seu desempenho pedagógico, processo de adaptação e evolução no contexto escolar (quando for o caso). Esse atendimento também propicia oportunidade de perceberem as capacidades\potencialidades e as dificuldades de seus filhos. Ademais, orienta a escola, coordenadores e professores, se necessário.

As atividades da psicopedagogia no CEIR busca estimular o desenvolvimento global da criança, adaptando, integrando e socializando esta ao meio. Trabalha a introdução dos conceitos pedagógicos do concreto ao simbólico, desenvolvendo o pensamento simbólico, proporcionando reconhecimento de imagens e enriquecimento do vocabulário. Estimula a leitura, escrita e raciocínio lógico 


\section{conghESSO CIENCIAESOCIEDADE

matemático. Trabalha as dificuldades de aprendizagem no processo de alfabetização, analisando a estruturação do pensamento em relação a leitura e escrita.

De acordo com Scoz (2008, p. 72) "no processo ensino e aprendizagem as dificuldades estão presentes e não podemos ser omissos". Neste campo, o papel do psicopedagogo é de grande importância, pois um diagnóstico precoce ajuda de forma progressiva e precisa a criança a se inserir neste processo de querer e possuir conhecimentos, aprendendo a superar seus limites, através da abordagem psicopedagógica. Mediante esta citação salienta-se que a psicopedagogia tem o papel de diagnosticar o problema, intervir e agir de forma que a criança consiga perceber e ter vontade de se apropriar do conhecimento. Neste contexto, analisaremos as possíveis intervenções psicopedagógicas na dificuldade de aprendizagem da leitura e escrita.

De acordo com Weiss (2008, p.62) ao avaliarmos os alunos que apresentam dificuldades de aprendizagem, de leitura e escrita, vamos encontrar diversas categorias. Haverá aqueles que necessitam da "intervenção psicológica ou psicopedagógica, ou até mesmo, aqueles que o problema pode ser resolvido dentro do contexto escolar, por meio de programas individualizados de ensino e práticas pedagógicas diferenciadas". Dessa forma a avaliação torna-se um elemento muito importante para traçarmos o caminho a seguir. Avaliar não para classificar, para rotular, mas para promover alternativas.

De acordo com Azenha (2008, p.76), a psicopedagogia é a área do conhecimento que estuda como as pessoas constroem o conhecimento. Em outras palavras, busca decifrar como ocorre o processo de construção do conhecimento nos indivíduos. Assim, ela se propõe a identificar os pontos que possam, porventura, estar travando essa aprendizagem, atuar de maneira preventiva para evitá-los e, ainda, propiciar estratégias e ferramentas que possibilitem facilitar esse aprendizado.

Quanto às ações práticas da psicopedagogia é necessário que sejam esclarecidos os motivos de sua aplicabilidade, e quais os devidos recursos a serem utilizados em cada momento, para que esteja ao conhecimento de cada criança o motivo que a leva a executar cada ação, seja com brinquedos ou com jogos. 


\section{CONSIDERAÇÕES FINAIS}

Em relação ao atendimento psicopedagógico no CEIR, pode-se concluir que objetiva o resgate de aspectos bloqueados do processo de aprender e, consequentemente, desenvolve a aprendizagem significativa e prazerosa, considerando a singularidade de cada criança. É caracterizado, de fato, que é de grande valor a utilização de brincadeiras e de jogos como metodologias de ensino dentro do processo de desenvolvimento da aprendizagem e que o psicopedagogo deve utilizar essas ferramentas desde os primeiros diagnósticos, nos levantamentos de hipóteses, para buscar uma melhor compreensão do nível de aprendizagem das crianças e também sobre quais as formas que cada uma pode aprender melhor, além de vislumbrar junto a cada família as possibilidades reais de inclusão, seja no acesso a escola com matricula regular, permanência, e desconstrução de paradigmas que a deficiência é limitante.

Desta forma, entende-se que o psicopedagogo nesta instituição trabalha em uma relação de troca, com seu papel definido e experiência dentro do imenso universo de ações que é a educação. O psicopedagogo no Centro de alta complexidade atua em razão das dificuldades encontradas na aprendizagem, causadas, especialmente, por problemas na aprendizagem da escrita ou da leitura, auxiliando na reabilitação das crianças com deficiência, visualizando potenciais até então ocultados pelas limitações físicas e cognitivas. Desafios e possibilidades estão na esteira desse processo, mas existem trilhas abertas seja com inovação, compromissos, construção de possibilidades e a certeza que o passado de exclusão tem que ser reconstruído, refeito à luz de vontades.

ABSTRACT: Through learning, the subject is inserted in a more organized way in the cultural and symbolic world that embodies society. This article aims to analyze the contribution of psychopedagogical care in the rehabilitation and habilitation of children with disabilities in the Integrated Rehabilitation Center (CEIR), in Teresina - PI. Its specific objectives are: to demonstrate learning from the psychopedagogical perspective, to understand the psychopedagogical work in a High Complexity Center and; cite strategies used in the psychopedagogical care of CEIR. The methodology was based on a qualitative bibliographic and documentary study and a descriptive and 


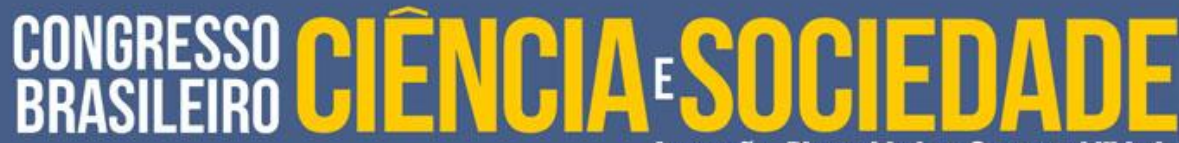 \\ -}

explanatory approach, with the inclusion of information published by CEIR. It is concluded that psychopedagogy plays a relevant role in the socialization of available knowledge, promoting the cognitive development of the learner and making him more independent and autonomous, considering its limitations.

Keywords: Psychopedagogy. Deficiency. Rehab center.

\section{REFERÊNCIAS}

AZENHA, L. D. T. Atuação psicopedagógica e aprendizagem escolar. Petrópolis: Vozes, 2008.

BOSSA, Nadia A. Dificuldades de Aprendizagem: O que são? Como tratá-las? Porto Alegre: Artmed, 2000.

CHAMAT, Leila Sara José. Técnicas psicopedagógicas: o diagnóstico clinico na abordagem interacionista. São Paulo: Vetor 2014.

FERNANDEZ, Alícia. O Saber em jogo: a psicopedagogia propiciando autorias de pensamento. Porto Alegre: Artmed, 2001.

FERREIRA, Fernandes Sisto. Dificuldades de aprendizagem no contexto psicopedagógico. Petrópolis, RJ: Vozes, 2010.

https://www.ceir.org.br/wpcontent/uploads/2017/12/relatorio de gestão. Acessado em 20.06.2019.

LÜDKE, Menga; ANDRÉ, Marli E. D. Pesquisa em educação: abordagens qualitativas. 2. ed. São Paulo: E.P.U., 2014.

PAIN, Sara. Diagnóstico e Tratamento dos Problemas de Aprendizagem. 4ạ ed. Porto Alegre: Artes Médicas, 2006.

RUBINSTEIN, Edith. A especificidade do diagnóstico psicopedagógico. In: Sisto, F. et al. Atuação psicopedagógica e aprendizagem escolar. Petrópolis: Vozes, 1996.

SCOZ, Beatriz. Psicopedagogia e realidade escolar: o problema escolar e de aprendizagem. 4aㅡ ed. Petrópolis, RJ: Vozes. 2008.

VYGOTSKY, Levi Seminovichi. Psicologia Pedagógica. Trad. Claudia Schilling. Porto Alegre: Artmed, 2001.

WEISS, M. L. Reflexões sobre o diagnóstico psicopedagógico. Porto Alegre: Artmed, 2008. 